\title{
LOS PRIMEROS BAJACALIFORNIANOS: ENIGMAS CRONOLÓGICOS, ECOLÓGICOS Y SOCIOCULTURALES*
}

\author{
Por \\ Eric W. Ritter**
}

\begin{abstract}
RESUMEN
Lainvestigación sobre los primeros pobladores de Baja California está aún en la infancia, rodeada de emoción y controversia. La hipótesis más aceptada considera que la gente del complejo arqueológico San Dieguito ${ }^{1} \longrightarrow$ parte de la cotradición lítica occidental- entró por el norte de la península hace 9,000 a 10,000 años aproximadamente.

En la búsqueda de los primeros hombres, la Laguna Seca Chapala y la costa noroeste del estado de Baja California han recibidola mayor parte de la atención arqueológica. El autor de este trabajo habla de las investigaciones realizadas intentando hacer inferencias sobre la vida de los primeros pobladores en base a la evidencia más confiable.
\end{abstract}

\begin{abstract}
The search for the first peoples of Baja California is still in its infancy, beset by both excitement and controversy. The most widely accepted hypothesis has San Dieguito peoples, part of the Western Lithic Cotradition, entering the peninsula from the north about 9,000 to 10,000 years ago.

Laguna Seca Chapala and the northwest coast of the State of Baja California have recieved the most archaeological attention in the state in search for early humans. The author of this article speaks of the research done trying to infere from the most reliable evidence aspects on the life of the ancient people.
\end{abstract}

\section{INTRODUCCIÓN}

En Baja California, la búsqueda de los primeros pobladores -0 al menos de la antigua presencia humana- y de la definición de sus expresiones socioculturales y adaptativas ha intrigado a los investigadores desde principios del siglo XX. Esta búsqueda ha estimulado una cantidad de investigaciones arqueológicas, algunas excavaciones y la siempre presente controversia y el debate.

* Traducido del inglés por Julia Bendímez y Diana Guerrero González.

** Arqué́logo, labora en el Departamento del Interior, Oficina de Territorialidad.

1 N. del E. Complejo arqueologico de una antigüedad de 8,000 a 10,000 años que se identifica en el sur de California, suroeste de Arizona y porciones de Baja California. 
En este documento se presenta una breve revisión de los hallazgos arqueológicos relacionados a los tempranos moradores de la península enfocándose en una de las áreas más estudiadas, la Laguna Chapala en el estado de Baja California, México. La visión que aqú se expresa acerca de la controvertida pregunta sobre la temprana incursión humana a la península (esto es, hace más de 8,000 afios) es conservadora, cautelosa y basada en el cuestionamiento; es más, en relación con este tema antropológico, se ofrece una breve discusión acerca del origen, organización social y ecología humana de los primeros habitantes de la península. Esta aproximación está fuertemente influenciada por una postura que enfoca la interacción del hombre con el medio ambiente; el medio ambiente visto como ambos: natural y cultural. También debe señalarse que mucho del trabajo hecho en Baja California sobre sus primeros habitantes debe ser examinado críticamente a la luz de los descubrimientos realizados en el norte: en el sur de California, en la Gran Cuenca, en el sur de los Estados Unidos y más allá, donde se ha mostrado más atención científica hacia este aspecto y de donde sin duda provienen los antiguos moradores de la península.

\section{ESTUDIOS CONTROVERTIDOS}

Engerrand $(1913)^{2}$ fue uno de los primeros científicos que indagó sobre la presencia de los primeros habitantes de Baja California. Trató lo concerniente a los denominados "pseudo-eolitos", ubicados en la zona noroeste de la península, e indicó que podían ser confundidos como evidencia del "hombre fósil". Más recientemente, Massey (1947:352), en una discusión sobre los hallazgos realizados en el oeste del Arroyo de Comondú en Baja California Sur, comenta que "huesos de bisonte seccionados a lo largo y huesos reportados como de camello y caballo, con extremos calcinados, son indicios del trabajo de humanos contemporáneos". Sin embargo, la ausencia de restos culturales asociados y la interrogante acerca de los orígenes de los huesos arrojan dudas acerca de la posible asociación humana. ${ }^{3}$ En esta área se requiere de más trabajo.

Cerca de la frontera de México con los Estados Unidos ha habido una serie de descubrimientos controvertidos relacionados con restos culturales de supuesta gran antigüedad. Childers y Minshall (1980) reportan un sitio localizado en el Arroyo Yuha-Pinto que según ellos contiene "herramien-

2 Su trabajo fue traducido acompañado de una introducción de Paul Ezell, 1981.

3 Confróntese a: Shipman (1981), Binford (1981), Behrensmeyer et al. (1986) y Kreutzer (1988). 
tas de piedra lasqueada y lascas que presentan huellas de uso". Estos autores proponen una fecha de más de 50,000 años para estos hallazgos basándose en fechamientos de radio carbono 14 , de restos de tufa de agua dulce y de concha de moluscos de una unidad geomorfológica relacionada. Estos autores han visto in situ, durante su descubrimiento, varios de los artefactos reportados y existe poca duda de que por lo menos algunos de ellos provienen de contextos geológicos del holoceno/pleistoceno. Pero la pregunta más importante es que si estos objetos de piedra fueron, de hecho, manufacturados por humanos o son producto de la naturaleza (cf. Payen, 1982). También para este asunto se requieren más estudios.

A poca distancia de Yuha se descubrió el entierro de Yuha, que originalmente fue considerado de una antigüedad de más de 20,000 años (Bischoff, et al. 1976). El trabajo subsecuente (Payen et al. 1978, 1979; Stafford, et al. 1984) mostró que los restos fúnebres eran más recientes, aproximadamente de 2,000 a 4,000 años de antigüedad. El descubrimiento original y el primer reporte con fecha muy antigua estimuló a José Luis Lorenzo, del Instituto Nacional de Antropología e Historia, para que excavara un montículo de piedra que se ubicaba en el lado mexicano de la frontera, en la búsqueda de un entierro de gran antigüedad; sin embargo, aparentemente no hizo descubrimiento alguno (Morlin Childers, comunicación personal, 1977). Una excavación similar, llevada a cabo en 1979 por Jay von Werlhof del Colegio del Valle Imperial en El Centro, California, efectuada en un montículo de piedra del lado estadounidense de la frontera y a corta distancia de Ocotillo, California, también tuvo resultados negativos (Jay von Werlhof, comunicación personal, 1979). Wilke (1978) ha observado que los entierros compuestos de montículos de piedra en los desiertos del sur de California (y por ende extendiéndose en el norte de Baja California) datan de 3,000 años A.C., ya de épocas históricas.

George Carter, en publicaciones de 1957 y 1980 (y en otros trabajos), ha sido un fuerte defensor de la existencia de restos arqueológicos del paleolítico inferior (de hace 80,000 a 100,000 afios) en la zona de San Diego, California (que presumiblemente también incluya la región de Tijuana). Sus estudios y conclusiones han sido secundados por Moriarty (1972) y Minshall (1976). Por otra parte, Reeves (1985) ha propuesto un estudio actualizado de los sitios localizados en la región del sur de San Diego para cuestionar los fechamientos y algunas de las categorías de artefactos establecidos por Carter. No obstante, él cree haber encontrado evidencia de ocupación durante mediados de la era Wisconsin. Berger y Huntley (1986) manifiestan que hay que tener precaución con algunas de las fechas que propone Reeves, pero consideran creíble que el material que fue directamente fechado, data de aproximadamente 14,000 años antes del 
presente, sin importar su origen, natural o cultural; no ofrecen comentarios sobre la validez de las intrepretaciones orientadas a lo cultural. La discusión y crítica de Moratto (1984:59-62) sobre mucho del trabajo que se menciona aquí (excepto el de Reeves) cuestiona el estatus arqueológico de los sitios. Los resultados obtenidos por Reeves deben esperar y ser sometidos a la discusión y a la crítica de las fechas. Por tanto, las implicaciones acerca de la prehistoria de Baja California deben permanecer en la incertidumbre.

En 1977, Morlin Childers habló sobre una tecnología lítica antigua denominada "ridge-back", que se localiza en la porción peninsular del desierto del Colorado. Al examinar algunos de estos objetos de piedra, este autor cree que son una mezcla de pedruscos erosionados por el viento (vientofactos) y herramientas de piedra lasqueada, probablemente de edad desconocida.

Por otro lado, Alan Olson, de la Universidad de Denver (comunicación personal, 1971), reportó haber observado grandes herramientas de piedra lasqueada cubiertas con gruesos depósitos de caliche en la región de las Tres Vírgenes en Baja California Sur. El caliche, sin embargo, se forma a diferentes velocidades (cf. Marion, 1989) debido a una variedad de factores, pero puede ser fechado si esas variables (tales como el cambio climático, fuente original del material, contribucion eólica, etc.) pueden ser controladas. Trabajo adicional en este tema puede ser prometedor para la definición de la antigüedad de las sociedades humanas primitivas de la península.

\section{DEFINICIÓN DE EXPRESIONES CULTURALES PRIMITIVAS}

Uno de los verdaderos pioneros de la arqueología del norte de Baja California fue Malcolm Rogers $(1939,1945,1966)$ quien ayudó a definir las primeras secuencias culturales del sur de la alta California y del norte de Baja California. Sus descubrimientos permanecen como la piedra angular en la delimitación y definición de los arreglos de artefactos humanos más antiguos aceptados por los estudiosos. Las tradiciones culturales antiguas de la porción occidental de California, definidas por Rogers (ver también a Moratto, 1984:76), incluyen sitios tierra adentro, en las playas de antiguos lagos y esteros o en zonas costeras, a lo largo de los márgenes de canales de viejas corrientes y estuarios.

Los conjuntos arqueológicos de estos sitios se caracterizan por pesadas puntas de proyectil de piedra que se insertaban en dardos o en lanzas cortas; pero con poca o nula existencia de instrumentos de molienda en roca fija, un surtido de implementos especializados como núcleos, lascas, navajas, guijarros; crecientes de piedra lasqueada, un surtido de raspadores, herramientas similares a cuchillos, martillos y buriles. 
Una de las tradiciones antiguas más difundidas en el desierto del occidente es la tradición de piedra estriada (Moratto, 1984:79) que se caracterizan por las puntas grandes y acanaladas, tipo de las culturas clovis y folsom que fueron utilizadas para la cacería de grandes herbívoros, actualmente extintos en su mayoría. Una punta de tipo clovis fue reportada por Aschmann (1952) procedente de un lugar cerca de San Ignacio, a la mitad de la península. Tal suceso aislado cuando mucho sugiere una ocupación efímera de cazadores de grandes especies; pero, también es posible que este artefacto haya sido transportado desde otra parte como una curiosidad, o puede tratarse de un artículo pseudo-clovis accidentalmente manufacturado. Una evidencia sólida de la tecnología clovis-folsom es escasa en la península.

Lo que ha sido denominado cotradición lítica de oeste (Davis, et al., 1969), que data de 11,000 a 8,000-7,000 anfos, presenta mejor evidencia para Baja California. Rogers $(1939,1945,1966)$ anteriormente le denominó a esta tradición "San Dieguito", algunos investigadores sobre el norte de Baja California prefirieron también este término (Treganza, 1942,1947; Massey, 1947; Warren, 1967). Rogers (1966: 91,99,184) identificó seis sitios ubicados en la costa del Pacífico en el norte de Baja California, desde el Rancho Johnson en el sur hasta el río Rosario en el norte, cuyo origen se atribuyó a San Dieguito.

En un sitio particularmente importante (LC-26) cerca de El Descanso, y conocido como "La Fábrica", se encontró una herramienta creciente 4 que es el ejemplar de su tipo que más al sur se ha encontrado (un instrumento también encontrado en conjuntos de puntas de tipo folsom y clovis).

Davis, Brott y Weide (1969) separan las expresiones provenientes de la costa de aquéllas provenientes de tierra adentro, y postulan una tradición paleo-costera como elemento de la cotradición lítica de oeste. Como lo observa Moratto (1984:104), la discusión se basa más en diferencias económicas que tecnológicas (adaptaciones costeras versus lago-marisma de tierra adentro), este autor lo ve meramente como diferentes enfoques de grupos relacionados. De hecho, como es evidente en la discusión que se da a continuación, en Baja California tanto los grupos de tierra adentro como los de la zona de la Laguna Chapala, bien pudieron haber explotado la costa en una forma estacional o periódica. También pudo haber sido el caso de áreas del sur de la alta California; es más, Rogers (1966:24) ha definido una fase del suroeste, que incluye la parte occidental no montañosa del

\footnotetext{
4 Creciente es un instrumento de piedra lasqueada en forma de luna en cuarto creciente cuya función aứn se desconoce.
} 
estado de Baja Califomia y todo Baja California Sur; así como una fase del centro que incorpora la parte baja del desierto del Colorado del estado de Baja California.

Rose Tyson, del Museo del Hombre (de San Diego, Ca.) ha presentado información sobre las excavaciones efectuadas en la cueva Baldwin en Punta Negra en la costa central del lado del Pacífico de Baja California, cuyas pruebas efectuadas de radio carbono 14 indican tener una antigüedad de hace más de 8,800 años antes del presente, y se remontan a épocas prehistóricas tardías (cf. Linick, 1977:30). Los resultados de este estudio todavía no han sido publicados y los nexos culturales son desconocidos; sin embargo, este descubrimiento puede corresponder al fechamiento firme más antiguo, asociado a restos culturales en la península, y que pudiera dar soporte para pensar en una ocupación costera San Dieguito hasta la parte baja de la costa del Pacífico de Baja California.

\section{LAGUNA SECA CHAPALA: ESTUDIOS ANTERIORES}

Búsquedas recurrentes en pos de restos humanos antiguos se han centrado en la laguna Chapala y en menor grado en las cercanías de la laguna Guija y laguna Amarga (ver figura 1). William Massey (1947:354) fue el primer investigador que reportó materiales sobre San Dieguito. Posteriormente, Brigham Arnold, un colega de Massey en la Universidad de California, completó los estudios detallados de la región de la laguna Chapala $(1957,1971,1984)$ que de una forma insistente proponian una muy antigua presencia del ser humano en esa área. Emma Lou Davis (1968) siguió los pasos de Arnold, y definió dos sitios de filiación paeloindia en la cuenca este del lago. Este autor (Ritter, 1976, 1979a y 1979b; Ritter et al. 1978, 1984), estimulado por el trabajo de todos los estudiosos mencionados, completó los estudios de la región.

Los restos culturales obtenidos en estas investigaciones, mientras no estén firmemente fechados, sugieren ambas situaciones: tanto ocupación reciente como antigua. La interrogante no sólo se refiere a la antigüledad, sino también sobre el comportamiento y el proceso: ¿Cuáles son las implicaciones del fechamiento de laguna Chapala en cuanto a la penetración inicial humana en la península?, ¿Cuáles son las respuestas de adaptación?, ¿Cómo estaban organizados socialmente los grupos y qué procesos sociales se dieron en los modos de vida en la medida en que el ambiente y la cultura cambiaron? Estas preguntas serán depejadas después de un breve examen de la evidencia arqueológica y varias opiniones y preguntas sobre esta información. 
Un problema significativo en la diferenciación de complejos arqueológicos tempranos (e.g. paeleoindígena/San Dieguito) de aquéllos que son de origen más tardío es el efecto de palimpsesto; artefactos encontrados en la superficie o próximos a la superficie que son remanentes de una ocupación recurrente y uso de materiales donde no hay acumulación de depósitos. Los complejos más antiguos sólo han sido ubicados cronológicamente por medio de correlaciones de fechas de éstos con los complejos (grandes puntas de proyectiles primarios de la serie que presentan forma de tallo) de regiones cercanas de los Estados Unidos. Sin embargo, Arnold 1984:27) observa que la utilización humana más temprana en la laguna Chapala se presentó durante, o antes, de la antigua época en que el lago presentaba niveles altos y fue representada por ciertos tipos de artefactos (bifaciales grandes, alargados) generalmente no aceptados como diagnósticos de tiempo.

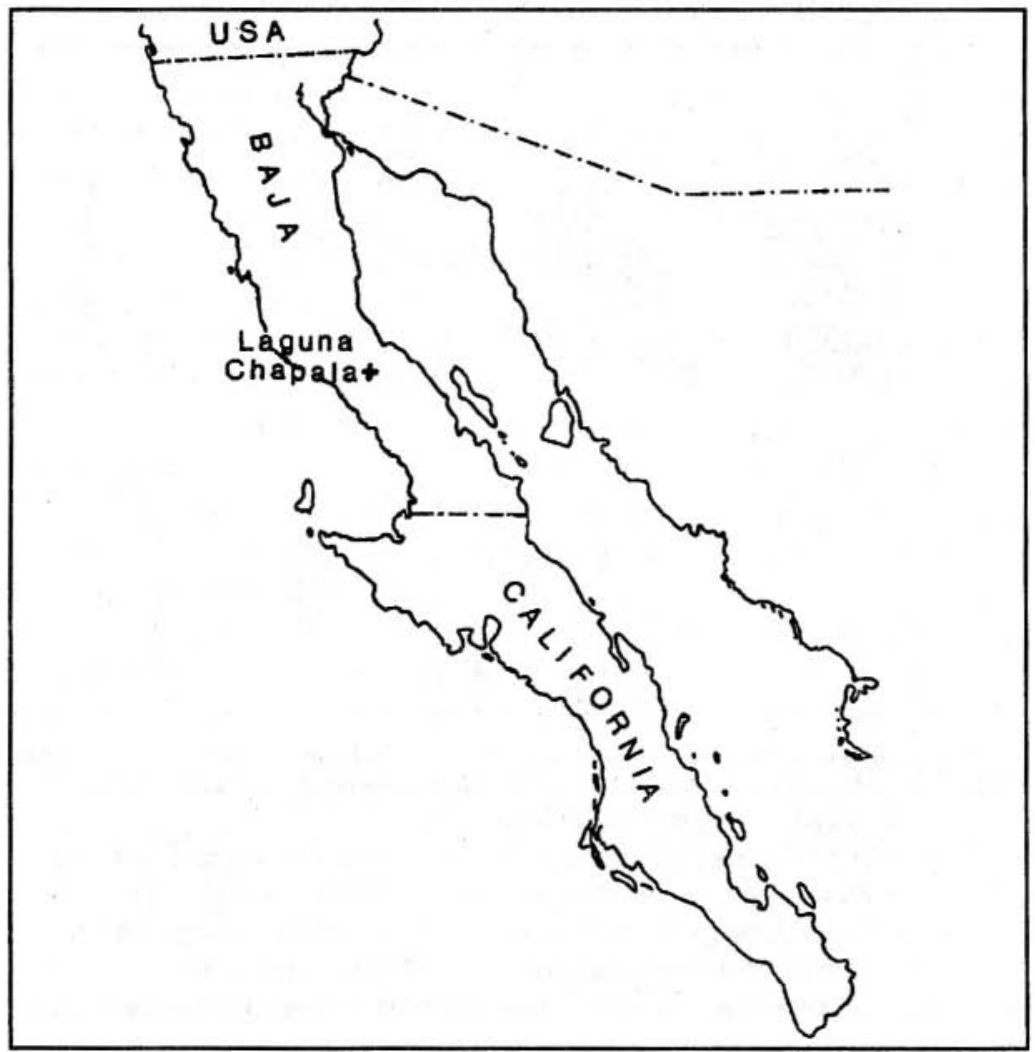

FIGURA 1. Ubicación de la laguna Chapala en Baja California. 
Ritter (1976) y Arnold (1984) de alguna manera tienen diferentes apreciaciones acerca de la naturaleza de la evidencia. La controversia se centra en: la función y en la ubicación cronológica de los instrumentos bifaciales alargados y de los artefactos que aparentemente están asociados; la significancia de la intemperización y la calidad artesanal de los bifaciales y la naturaleza de la distribución en sitio frente a características geomorfológicas. No hay desacuerdo en torno a la evidencia de los niveles anteriores del lago, probablemente controlados por el clima y por los movimientos tectónicos, ni parece haber desacuerdo entre los varios arqueólogos que han trabajado en esta cuenca en cuanto a que la presencia paleoindia o San Dieguito se ubicaba en la actual orilla del lago, como es evidenciado por los artefactos San Dieguito, que incluyen puntas de proyectil semejantes a los del lago Silver y lago Mojave (cf. Warren y Crabtree, 1986:184-185) (ver figura 2). La pregunta se centra realmente en la ocupación del pleistoceno: ¿Hay evidencia de ella?

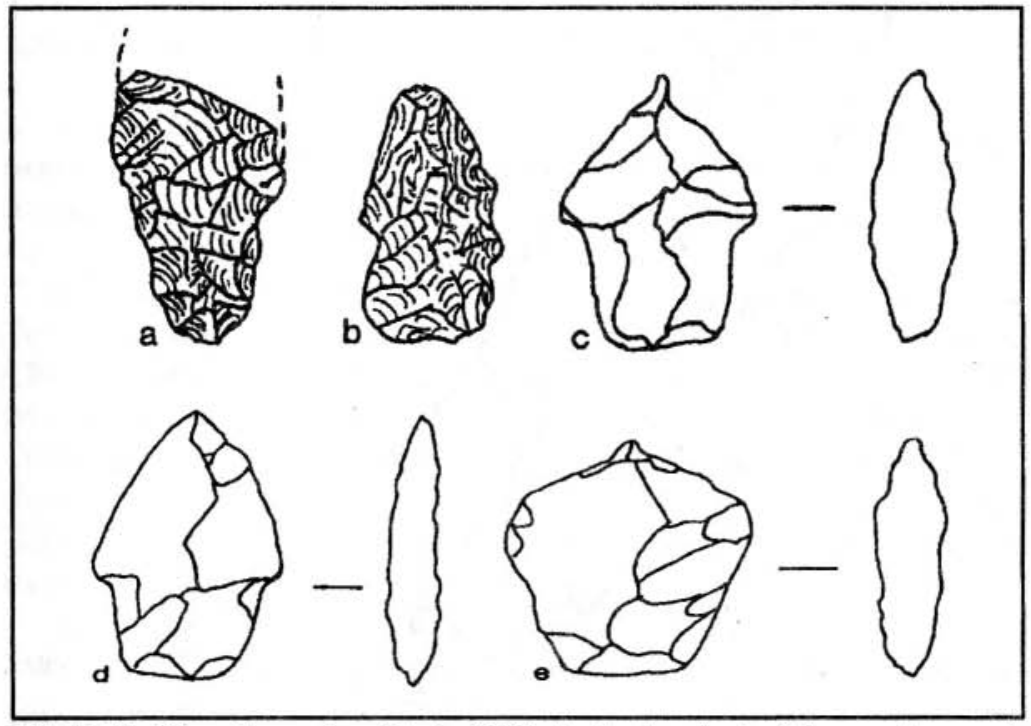

FUENTE: Véase Emma Lou Davis, 1968. An Archaeological Reconnaissance in the Central Desert of Baja California.

a. Punta de proyectil tipo Lago Mojave de petrosílice (longitud de $4.25 \mathrm{~cm}$.).

b. Punta de proyectil de cuarzo, tipo Lago Silver (el largo es de $4.2 \mathrm{~cm}$.).

c. Punta de proyectil del tipo de Lago Silver-Mojave (largo 3-4 cm.

d. Una punta de proyectil estilo Lago Mojave (largo $4-6 \mathrm{~cm}$.)

e. Una punta de proyectil tipo Lago Mojave (largo aproximado $5 \mathrm{~cm}$.)

FIGURA 2. Puntas de proyectil encontradas en la laguna Chapala. 
Los problemas mencionados anteriormente no pueden tener solución sin el uso de mejores métodos de análisis; por ejemplo ¿los artefactos bifaciales alargados (ver figuras 3 y 4 ) son realmente instrumentos, o son el resultado de una fase inicial de la manufactura de herramientas 0 ambas cosas?

¿La distribución espacial de estos objetos es resultado de su uso o de su transporte desde otros sitios, de núcleos o preformas, o las dos cosas como piensa el autor? ¿Su presencia en los niveles altos del lago y en unidades aluviales/coluviales implica una edad más antigua, o su ubicación es una mera coincidencia, resultado de que tales sitios eran lugares en donde se trabajaban las primeras fases de la creación de la herramienta ubicados cerca de sitios de materia prima, o son posibles los dos escenarios? ¿Cómo asignar edad a los grandes bifaciales basándonos en el grado de pátina y enrojecimiento provocado en la piedra por arcillas, otros minerales y microorganismos (donde el artefacto ha estado yaciendo en el suelo)? ¿Por qué algunos bifaciales de tamaño mediano con similar descomposición y enrojecimiento son encontrados en sitios próximos al actual lecho del lago? La opinión de este escritor, como se menciona arriba, es la de que fueron transportados a la actual orilla del lago y provienen de los estratos más elevados de la cuenca poco después de su manufactura. Las respuestas al fechamiento de ambos artefactos y al perfil del suelo deben estudiarse más a fondo.

Dorn (1984) y Dorn et al. (1986) han aplicado los métodos de fechamiento "radio-cation" y radiocarbono-acelerador para barniz de roca en ambos artefactos y en pendiente de superficies, una técnica prometedora para fechar artefactos de superficie de la totalidad de la península. El trabajo de Nanson y Young (1987) es prometedor para fechar objetos asociados a depósitos aluviales y a través del fechamiento termoluminiscente. McFadden et al. (1989) plantean otros medios para calcular las edades relativas de los abanicos aluviales del desierto, como es el de un examen del tipo de suelo y del desarrollo de la variabilidad del abanico, acumulación de barniz, y enrojecimiento de las partes posteriores de las rocas superficiales. Entre paréntesis, Dorn (1988) encontró que el proceso de formación de un barniz incipiente y de los primeros indicios de enrojecimiento en las caras de abajo de las rocas superficiales, en el valle de la Muerte, es de aproximadamente 2,000 af́os. Con una cierta cantidad de artefactos de la laguna Chapala que exhiben considerable enrojecimiento (dejando de lado las diferencias ambientales regionales) quizás entonces uno pudiera estimar que, sin duda, muchos de estos artefactos, incluyendo los bifaciales alargados, son algo antiguos, tanto los próximos a la actual orilla del lago como los que están más alejados. 
Evidentemente, análisis geomorfológicos detallados (en relación con el trabajo de Arnold) están garantizados en la laguna Chapala, de acuerdo con los estudios arqueológicos que incorporan muchas de las nuevas técnicas y métodos que se han desarrollado desde el esfuerzo pionero de Arnold. Sobre lo anterior también el Dr. Arnold (1984:34-35) hace un planteamiento similar.

La antigüedad de los artefactos de la laguna Chapala es controvertida, ya que hay restos culturales que datan desde, por lo menos, el temprano holoceno hasta la prehistoria tardía. Además de los bifaciales alargados -y ovalados- (ver figuras 3 y 4 ) que trascienden varios periodos culturales, hay otros objetos de la cuenca (y de la cercanías de la laguna Guija) que aparentemente datan del holoceno temprano. Estos incluyen puntas de proyectil similares al estilo Lago Mojave y Lago Silver (ver figura 2) que han sido reportadas por Davis (1971) y Ritter (1979a:202-203), desde zonas tan al sur como la región de bahía de Concepción; sin embargo, hay alguna duda respecto a la similitud tipológica. El problema de asociar específicamente otros tipos de herramientas con los de la ocupación paleoindia o San Dieguito es muy difícil en términos de ambos, tanto el hecho de que un sitio se identifique con varias culturas, como se mencionó, como la continuidad de tipos de herramientas a lo largo del tiempo (cf. Laylander, 1987:444-448). Otros artefactos de piedra posiblemente tempranos (ver Davis, 1968 y Ritter, 1979a) incluyen grandes planos convexos, pequeños planos raspadores, bloques de golpeo, bifaciales (cf, Warren, 1966: láminas 16 y 17; Warren y Ranere, 1968:14), raspadores de extremo amplio, planos puntiagudos, marcados de rojo, buriles y raspadores laterales. Los restos de conchas son frecuentes en todos los sitios, sugiriendo un uso prolongado de estos productos, probablemente más como herramienta o recipientes que como alimentos. El sólo hecho de tratar de saber cómo fueron usadas en laguna Chapala las conchas más antiguas es problemático.

El trabajo arqueológico en las proximidades de laguna Guija, efectuado por el autor y otros (Ritter et al. 1978, 1984), da forma a una interesante base comparativa. Mientras que unos cuantos huesos de animal fosilizados fueron encontrados en la playa, ninguna asociación cultural era evidente. Se estima que casi todos los indicios de utilización humana (tanto en la orilla del lago como lejos del lecho del lago) son de la prehistoria tardía. La excepción es el descubrimiento de una o dos puntas de proyectil en el lecho del lago que parecen ser del tipo lago Mojave que por su forma sugiere su uso durante el holoceno temprano (cf. Ritter et al. 1978: fig. 1h). Es más, ninguno de los grandes bifaciales ovalados fueron encontrados en el lago. 

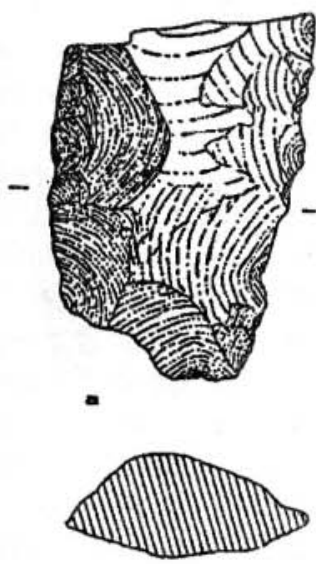

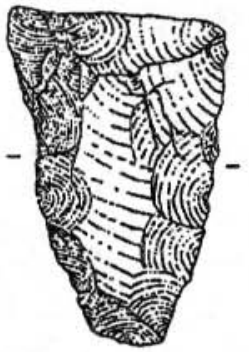

b

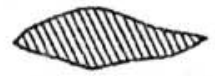

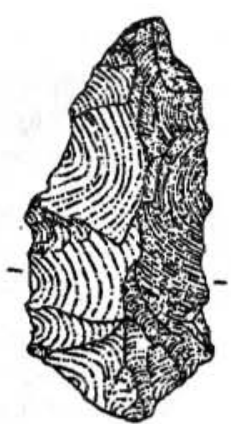

a

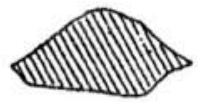

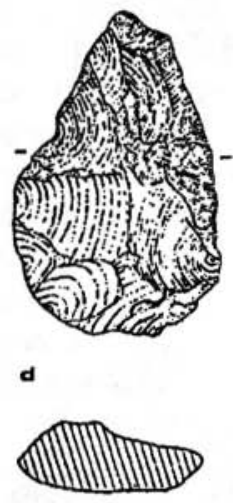
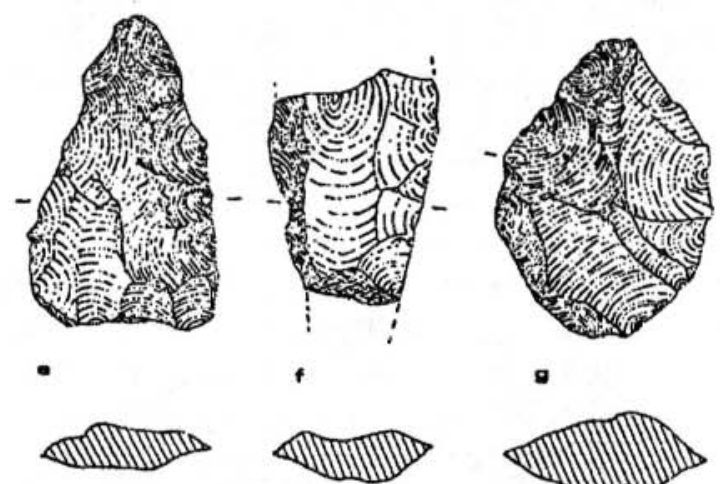

g
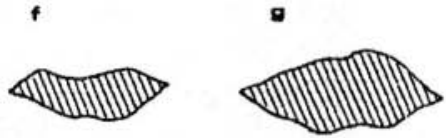

a. Bifaciales alargados de laguna Chapala (cerca del actual lecho del lago)

b. Bifacial de petrosílice (catálogo \#53-476). Largo: $6 \mathrm{~cm}$.

c. Bifacial de patrosílice (catálogo \#53-616). Largo: $5.6 \mathrm{~cm}$.

d. Bifacial de patrosílice (catálogo \#53-484). Largo $5.5 \mathrm{~cm}$.

e. Bifacial de patrosílice (catálogo \#53-363). Largo $5.3 \mathrm{~cm}$.

f. Bifacial de basalto (catálogo \#53-399). Largo: según como está ilustrado es de $4 \mathrm{~cm}$.

g. Bifacial de felsita (catálogo \#53-542). Largo: $5 \mathrm{~cm}$.

FIGURA 3. Bifaciales alargados de laguna Chapala (cerca del actual lecho del lago). 

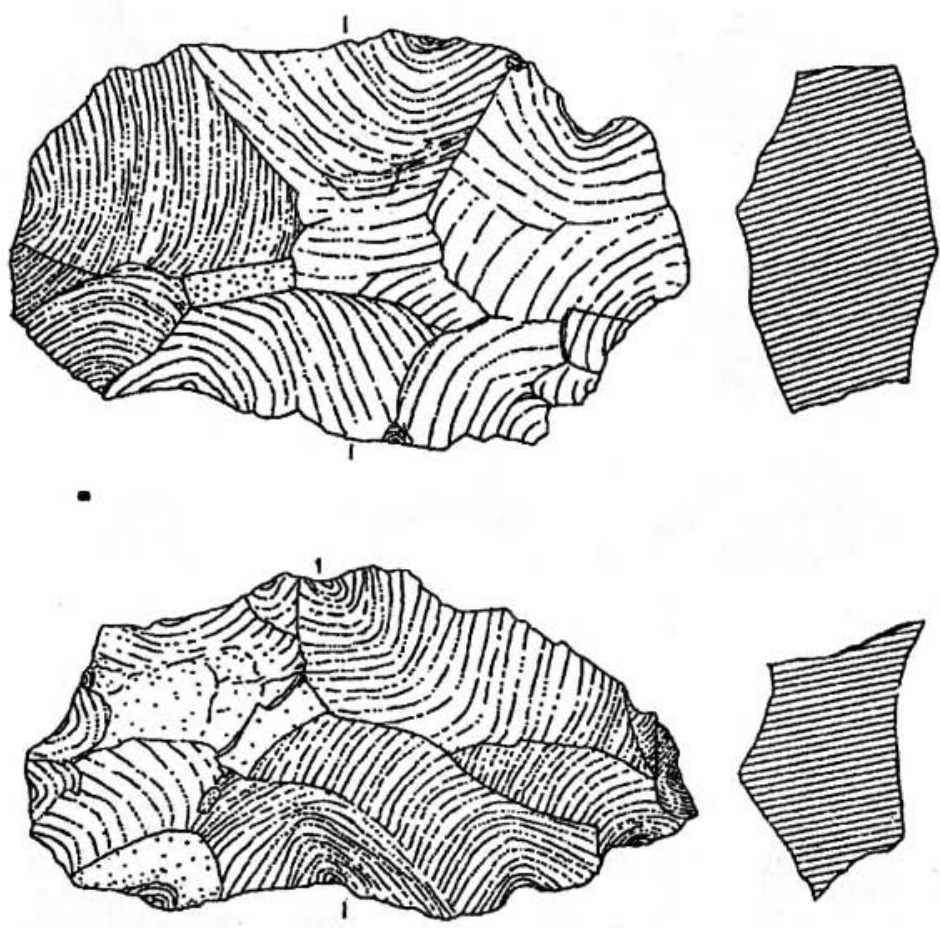

$\mathbf{b}$
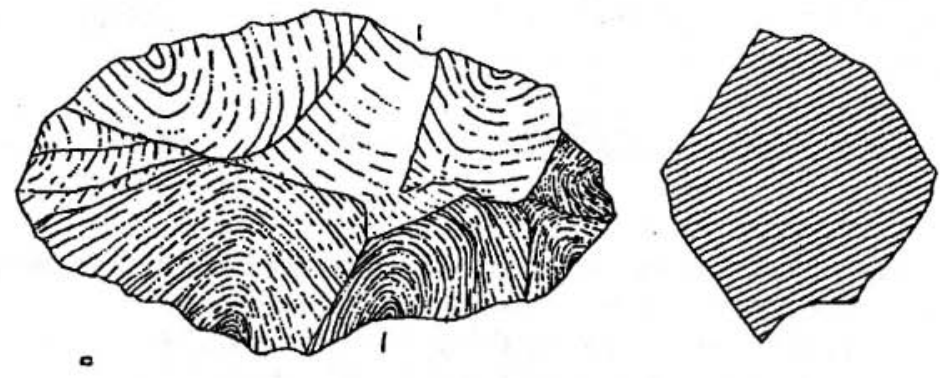

a. Bifacial de petrosílice (catálogo \#53-352). Largo: $11.5 \mathrm{~cm}$.

b. Bifacial de felsita. Largo: $11.4 \mathrm{~cm}$.

c. Bifacial de felsita.

FIGURA 4. Bifaciales alargados, laguna Chapala (orillas este). 
En la orilla oriental de la laguna Chapala se presenta lo que Amold considera el conjunto más antiguo (1957:25-253): el conjunto bifacial-ovalado. Artefactos de este conjunto fueron recuperados por Arnold en posiciones que están sobre o adyacentes a remanentes de niveles más elevados. Hay dos tipos de sitios, uno en el que los artefactos están asociados a operaciones de explotación de piedra, y el otro donde no existe tal asociación y los artefactos están empotrados en la superficie de las planicies marginales de grava. Grandes bifaciales alargados y ovalados están indudablemente presentes en gran cantidad en estos lugares, predominando en sitios de alta terraza/abanico (ver figura 4). Bifaciales más pequeños, del tipo San Dieguito, también fueron encontrados en varios sitios de estas orillas, pero son más frecuentes en la proximidad de las playas actuales.

Los efectos del medio ambiente y el enrojecimiento de los artefactos bifaciales y de otros objetos de petrosilice entre ambos sitios es inconcluyente. Las diferencias en pátina y en el enrojecimiento de los bifaciales y en otros artefactos de petrosflice, de ubicaciones más altas y más bajas en relación a la playa moderna, como se anotó, puede ser un reflejo tanto de la variación en la constitución química del suelo y de las diferencias en topografía (por el transporte de sedimentos abrasivos efectuado por el viento y por la exposición al medio ambiente), como de la edad. Es probable que instrumentos más refinados como bifaciales pequeños, erosionados (con pátina) y enrojecidos, fueran transportados inmediatamente de sitios de alta terraza/ventilados a campamentos más bajos a la orilla del lago, junto con especímenes más grandes que representaron desechos o escondites de artefactos sin terminar. Asimismo, poblaciones posteriores pudieron haber desechado o perdido artefactos en sitios más viejos y más elevados y haber transportado artefactos muy viejos de terrazas altas a sitios más bajos. Otros tipos de artefactos erosionados y enrojecidos presentes en sitios aledafios a la bahía sugieren actividades que se adicionan a aquéllas que podrían ser asociadas sólo con los bifaciales. La conclusión más plausible es que la ocupación o la actividad en los sitios elevados de terraza/ventilada era contemporánea, por lo menos durante el holoceno temprano, con actividades humanas específicas más próximas al actual lecho del lago. Otra conclusión razonable es la de que no hay aún una correlación directa de edades entre las más antiguas y más altas terrazas/ventiladas (esto es, un más viejo y alto nivel del lago) y los artefactos asociaciados.

\section{LAGUNA CHAPALA: INTERPRETACIONES ADICIONALES}

La proliferación de desechos de piedra lasqueada, de apariencia relativamente reciente, y de herramientas alrededor de las actuales playas de 
la laguna Chapala y laguna Guija, deja a uno con la impresión de que la actividad más frecuente, posiblemente por un número mayor de gente o por una ocupación más periódica, ocurrió cerca de la actual orilla de la playa durante tiempos arcaicos tardíos. Aquí también existe evidencia de la presencia humana tan temprana como desde pincipios del holoceno; $\mathrm{e}$ incluso materiales más antiguos bien pudieran estar enterrados cerca del actual lecho del lago, tal y como lo seffaló Arnold (1984:30). Artefactos erosionados y enrojecidos que aparecen más frecuentemente en formas terrestres más antiguas (abanicos y líneas costeras antiguas) sugieren a primera vista la existencia de actividades antiguas en lugares más elevados. Pero mientras aquí haya indicios que sugieren una ocupación tanto en el holoceno temprano así como actividades tardías, las diferencias en pátina y en enrojecimiento entre los sitios elevados y los bajos son probablemente, en lo fundamental, reflejo de las diferencias microambientales y de la variación en las etapas de reducción de herramientas de piedra y de los patrones de uso.

En resumen, en relación con la arquelogía del área de la laguna Chapala, el juicio de Davis es aún válido respecto de la inseguridad de la cronología e historia de la cultura:

...pero una repuesta aproximada podría ser que los sitios tierra adentro son paleoindígenas, del arcaico temprano y del arcaico tardío. La cultura paleoindígena (complejos sin utensilios de piedra para moler) aparece tecnológicamente relacionada con los complejos San Dieguito-Lago Mojave de Alta California (1968:200).

Es difícil arribar a una reconstrucción concreta de los patrones de asentamiento/subsistencia en la cuenca de la laguna Chapala, basándonos en los estudios arqueológicos y ambientales realizados a la fecha. Sin embargo, algunos comentarios generales e hipótesis de trabajo merecen ser considerados. Próximo al final del pleistoceno, o durante el holoceno temprano, pequeños grupos de cazadores y recolectores centraron sus actividades, por lo menos estacionalmente, en una serie de lagos pluviales ubicados a lo largo del oeste del desierto. Laguna Chapala fue uno de esos lagos. Condiciones lacustres únicas pero rápidamente cambiantes aumentaron el crecimiento y la diversificación de las plantas y atrajeron aves migratorias así como animales de cacería. El que esta megafauna, ahora extinta, reportada por Massey (1947:352) en las cercanías de Comondú y vista por este investigador en restos fósiles del lecho de la laguna Guija, fuera explotada es una cuestión digna de probarse por medio de excavaciones selectas en sitios dados y en determinadas formas terrestres. 
Además, el lago proveyó de agua dulce, aún en aquellas épocas pasadas que eran más húmedas, lo cual era una ventaja. Tales condiciones seguramente eran importantes y atractivas para esos recién llegados.

Bastante tiempo se tomaba en la localidad para explotar y, posiblemente, exportar o transportar a otras zonas trozos brutos de petrosílice y otras preformas y grandes bifaciales sin terminar. Adicionalmente, esos productos eran procesados para uso local. Estas piedras y talleres se encuentran principalmente en la orilla este, aunque una considerable manufactura de herramientas - cuando menos durante etapas y tiempos posteriores - se dio en sitios adyacentes al actual lecho del lago. Sin duda grupos de trabajo recorrieron un cierto número de kilómetros lejos de sus campamentos centrales ubicados a la orilla del lago, este es un patrón que se repite en tiempos prehistóricos tardíos.

Lo que sí es único en la arqueología del desierto occidental es el acceso y el uso, aparentemente temprano, de ambos productos, lacustres y marinos relacionados con su subsistencia, dentro de la ronda o ciclo estacional de un grupo, un aspecto que fue compartido sólo con otros grupos que aprovecharon los lagos interiores de la estrecha península de Baja California. Aparentemente, la ventaja adicional era la existencia de una fuente permanente y rica de alimentos marinos (pescado, moluscos, mamíferos marinos), limitada principalmente por el acceso al agua dulce y a alimentos vegetales suplementarios. Se presentó una menor fragmentación de los grupos, en respuesta a la escasez estacional, una mayor solidaridad y posiblemente una mayor exogamia (femenina) para estimular las relaciones con los grupos vecinos y para asegurar el flujo de información. Especulativamente, cada banda era posiblemente una unidad patriarcal de interacción y cooperación cerrada, compuesta de agregados familiares.

\section{INFERENCIAS RELATIVAS A LOS PRIMEROS HOMBRES DI BAJA CALIFORNIA}

¿Qué implicaciones tienen los resultados de los estudios realizados en la zona de la laguna Chapala, en particular, y la otra evidencia peninsular más confiable, en general, como soporte para inferir sobre el poblamiento inicial de Baja California y los aspectos socioculturales y de adaptación? Este es un asunto que se sustenta en escasísimas evidencias. Sin embargo, se ha hecho suficiente trabajo en América del Norte sobre las culturas tempranas para complementar los estudios hechos en Baja California. Asimismo, hay un sustancial cuerpo de información sobre las culturas de cazadores y recolectores de todo el mundo que provee una base para la interpretación; sin embargo, varios factores deben ser considerados. 
Hill y Hurtado (1989:457) han indicado que los grupos de cazadores y recolectores (el patrón que se presume corresponde a estos grupos antiguos) "varían en casi todos los parámetros en que se han medido: composición de la dieta, distribución de sus alimentos, patrones de trabajo de hombres y mujeres, sistemas de matrimonio, fertilidad y mortalidad". Además, uno no puede asumir que la cultura y el comportamiento del hombre de la prehistoria tenga análogos cercanos con los cazadores y recolectores de fechas recientes. Sin embargo, las comparaciones etnográficas con las inferencias basadas en la evidencia arqueológica, a través del uso de múltiples hipótesis de trabajo, pueden ser productivas.

Una conclusión es casi cierta, los primeros bajacalifornianos se enfrentaron a un ambiente físico muy diferente al actual. El fin del pleistoceno y del temprano holoceno fueron épocas de cambios ambientales. Era más fresco que hoy en día, con escasas comunidades vegetales, lagos y pantanos, y una fauna diversificada que pudo haber incluido bastantes ungulados, es decir los ancestros del borrego cimarrón, del venado y del antílope de tiempos recientes; y, posiblemente por un tiempo, grandes animales de cacería ahora extintos. Los patrones de la explotación de los recursos marinos eran quizás algo diferentes (cf. Fladmark, 1979:60-91), pero sin duda alguna la fauna marina todavía servía como una canasta alimenticia abundante, cuya captura requería de poca destreza, tales como mariscos y mamíferos marinos, donde los patrones de adaptación pudieron haber sido establecidos antes de, o al mismo tiempo, del cruce de Bering.

Los primeros cazadores de megafauna de norteamérica, enfocados al oeste en ambientes lacustres pero también utilizando los ecosistemas vecinos, parecen haber sido los usuarios de una tecnología de navaja y punta acanalada (11,000-13,000 affos antes del presente). Sin embargo, sólo se conoce una punta acanalada del tipo clovis en la península, y parece ser que, en base a información actualizada, se trató de gente postclovis, las del complejo San Dieguito-Lago Mojave, quienes fueron los primeros inmigrantes notables de la península.

Morrato considera (1984:88) que los de San Dieguito evolucionaron de gente que utilizaba puntas acanaladas (cuyos centros estaban en lagos pluviales y su hábitat era tipo pantano) hace aproximadamente 11,000 affos (ver también Aikens, 1978) y que subisitieron hasta hace 7,000 affos. Como se menciona antes, una concentración San Dieguito se encontraba presente durante este tiempo.

Una vez más, la laguna Chapala probablemente es la extensión más sureffa del foco caracterizado por los lagos pluviales de la cotradición lítica 
occidental. Sin embargo, las puntas tipo lago Mojave y lago Silver encontradas tan al sur como Bahía Concepción, en Baja California Sur, sugieren que esta gente se internó más al sur, posiblemente debido a su temprano enfoque marino.

Kelly y Todd (1988:233), a manera de hipótesis, han propuesto que a medida que el ambiente cambiaba, mientras el pleistoceno terminaba y daba inicio el cálido holoceno, se alteraba la distribución de recursos explotables. Los paleoindígenas podían haber enfrentado la reducción periódica de sus recursos de dos maneras: susituyendo un recurso agotado por otro en el mismo territorio, o cambiando territorios. Utilización humana del medio ambiente en tiempos tempranos en el norte de Baja California puede reflejar ambas respuestas, migración a la laguna Chapala y a otras cuencas vecinas y un nuevo o más intenso uso de los recursos costeros, o ambos.

\section{CONCLUSIONES}

Sin duda, las primeras poblaciones peninsulares eran escasas y se caracterizaban por una alta mobilidad logística y residencial sobre una amplia región (cf. Kelly y Todd, 1988:234-235). La unidad social y económica consistía probablemente de un grupo de varias familias extendidas. Los campamentos, como ilustran los de Baja California, parecen haber tenido vidas cortas, y la tecnología sugiere el uso de objetos de piedra portátiles y durables de materiales de buena calidad (e.g. petrosílice). Esto puede explicar también la alta seguridad en la industria bifacial (Kelly y Todd, 1988:237) y la ausencia de una bien establecida tecnología de molienda. Moratto (1984:78), sin embargo, afirma que "en algunas localidades favorecidas, tales como aquéllas cercanas a grandes pantanos y estuarios, la abundancia natural pudo haber sido adecuada para sostener asentamientos más o menos permanentes, y posiblemente así fue". La evidencia arqueologica en la laguna Chapala es muy escasa para apoyar esta idea.

A medida que el clima mejoraba, la población se incremento y en la medida que la biomasa faunística disminuía o la biomasa aumentaba y se volvía más aglomerada y confiable (como en las costas), los asentamientos pudieron haberse desplazado a las localidades costeras de uso estacional tierra adentro, en lagos y otras fuentes de agua. La estructura social tipo banda continuó o se afianzó, quizá en mayor grado en cuanto al sentido de territorialidad y de intercambio social en relación al matrimonio, intercambio de información, trueque, etc.

Evidentemente, hay muy poca información, relativamente hablando, sobre los primeros habitantes de Baja California. Los investigadores tienen algunas ideas sobre su tecnología y patrón de asentamientos, pero cuando 
los estudiosos profundizan en su organización social, ideología, y otras facetas culturales, están claramente en un escenario altamente especulativo. A pesar de factores de preservación y la carencia de trabajo arqueológico en la península, uno puede visualizar a esos grupos primitivos que van de nomádicos hasta asumir rondas estacionales programadas, probablemente con un alto nivel de flexibilidad. Estos pueden haber sido pequeños grupos multifamiliares que tenían contacto poco frecuente con otros grupos que se estaban interrelacionando con un ambiente bajo un flujo que creaba tensiones y cambios en la adaptación y, sin duda, en algún grado en la estructura social (posiblemente fragmentos de grupos familiares en algunas áreas y consolidación en otras). Una hipótesis digna de probar es que en tiempo del holoceno medio, las áreas interiores eran poco usadas, un patrón que persistió hasta tarde en la prehistoria, cuando adaptaciones en el desierto correspondían a una mayor adquisición de alimentos con regularidad, más fertilidad, bajos índices de mortalidad y aumento y dispersión poblacional concomitante.

Las respuestas a las muchas preguntas relativas a la temprana presencia humana en Baja California, a su estructura social y su comportamiento vendrán cuando se lleven a cabo estudios interdisciplinarios en localidades selectas. Desafortunadamente, el tiempo y las transgresiones humanas modernas están cobrando sus cuotas en esos restos. En pos de su mejoramiento, ¿pueden las sociedades de hoy esperar aún más para buscar y resolver los enigmas presentados por las sociedades de ayer?

\section{BIBLIOGRAFÍA}

AIKENS, C. Melvin. 1978. "The Far West". Jesse D. Jennings, (ed.). En: Ancient Native Americans, W.H. Freeman, San Francisco.

ARNOLD, Brigham A. 1957. "Late Pleistocene and Recent Changes in Land Forms, Climate, and Archaeology in Central Baja California". University of California Publications in Geography, vol.10.

- 1971. "The earlier traces of man in the two Californias". Baja California Symposium 9.

- 1984. "Early man in Baja California--evidence and interpretation". Pacific Coast Archaeological Society Quarterly vol. 20, no.1.

ASCHMANN, Homer. 1952. "A fluted point from central Baja California”. American Antiquity, vol. 17, no.3.

BEHRENSMEYER, A.K., K.D. Gordon, y G.T. Yanagi. 1986. "Trampling as a cause of bone surface damage and pseudocut marks". Nature. No. 319. 
BERGER, G.W. y D.J. Huntley. 1986. "San Diego research and chronology revisited". Current Research in the Pleistocene. Vol. 3.

BINFORD, Lewis R. 1981. Bones: Ancient Men and Modern Myths. Academic Press. Nueva York.

BISCHOFF, James L., Richard Merriam, W. Morlin Childers, y Reiner Protsch. 1976. "Antiquity of man in America indicated by radiometric dates on the Yuha burial site". Nature No. 261.

CARTER, George F. 1957. Pleistocene man at San Diego. The Johns Hopkins Press, Baltimore.

- 1980. Earlier than you think: A personal view of man in América. Texas A and M University Press, College Station.

CHILDERS, W. Morlin. 1977. "Ridge-back Tools of the Colorado Desert". American Antiquity. Vol. 42, no. 2.

CHILDERS, W. Morlin y Herbert L. Minshall. 1980. "Evidence of Early Man Exposed at Yuha Pinto Wash". American Antiquity.Vol.45, no. 2.

DAVIS, Emma Lou. 1968. "An Archaeological Reconnaissance in the Central Desert of Baja California". University of California, Los Angeles, Archaeological Survey Annual Report 1968. Vol. 10.

- 1971. "Ancient man in Baja California". The Masterkey. Vol. 45.

DAVIS, E.L., C.W. Brott, y D.L. Weide. 1969. "The Western Lithic Co-Tradition". San Diego Museum Papers. No.6.

DORN, R.I. 1984. "Geomorphological interpretation of Rock Varnish in the Mojave Desert, California". En: Surficial Geology of the Eastern Mojave Desert, California, J.C. Dohrenwend, ed. 1984 Annual Meeting Fieldtrip 14 Guidebook. Geological Society of America, Reno.

- 1988. "A Rock Varnish Interpretation of Alluvial-fan Development in Death Valley". National Geographic Research. Vol. 4.

DORN, R.I., et al. 1986. "Cation-ratio and Accelerator- radiocarbon Dating of Rock Varnish on Archaeological Artifacts and Landforms in the Mojave Desert, Eastern California". Science. No. 227. ENGERRAND, G.M. 1913. "Estado actual de la cuestión de los eolitos". Boletin del Museo Nacional de Arqueologla, Historia y Etnologia Vol. 2, no. 8.

EZELL, Paul H. 1981. "Report of an Excursion to Baja California by G. Engerrand". Pacific Coast Archaeological Society Quarterly Vol. 17, no. 1.

FLADMARK, K.R. 1979. "Routes: Altemate Migration Corridors for Early Man in North America". American Antiquity. Vol. 44, no. 1.

HILL, Kim and A. Magdalena Hurtado. 1989. "Hunter-gatherers of the New World". American Scientist. Vol. 77, no. 5. 
KELLY, Robert L. y Lawrence C. Todd. 1988. "Coming into the Country: Early Paleoindian Hunting and Mobility". American Antiquity. Vol. 53, no. 2.

KREUTZER, Lee Ann. 1988. "Megafaunal Butchering at Lubbock Lake Texas: A Taphonomic Reanalysis". Quarternary Research. Vol. 30 , no. 2.

LAYLANDER, Don. 1987. "Sources and Strategies for the Prehistory of Baja California". Tesis de Maestría, San Diego State University. LINICK, T.W. 1977. "La Jolla Natural Radiocarbon Dates, III". Radiocarbon. Vol. 19.

MARION, Giles M. 1989. "Correlation Between Long-term Pedogenic $\mathrm{CaC} 3$ Formation Rate and Modern Precipitation in Deserts of the American Southwest". Quarternary Research. Vol. 32, no. 3.

MASSEY, William C. 1947. "Brief Report on Archaeological Investigations in Baja California". Southwestern Journal of Anthropology. Vol. 3.

McFADDEN, Leslie D., John B. Ritter, y Stephen G. Wells. 1989. "Use of Multiparameter Relative-age Methods for Age Estimation and Correlation of Alluvial Fan Surfaces on a Desert Piedmont, Eastern Mojave Desert, California". Quaternary Research. Vol. 32, no. 3. MINSHALL, H.L. 1976. The Broken Stones. The Copley Press, San Diego.

MORATTO, Michael J. 1984. California Archaeology. Academic Press, San Diego.

MORIARTY, J.R. y H.L. Minshall. 1972. “A new pre-Desert site discovered near Texas Street". The Anthropological Journal of Canada Vol. 10, no. 3.

NANSON,G.C.y R.W. Young. 1987. “Comparison of thermoluminiscence and radiocarbon age-determinants from Late-Pleistocene alluvial deposits near Sydney, Australia". Quarternary Research. Vol. 27, no. 3.

PAYEN, Louis A. 1982. "The Pre-Clovis of North America: Temporal and artifactual evidence". Tesis doctoral, University of California, Riverside.

PAYEN, Louis A. et al. 1978. "Comments on the Pleistocene age assignment and associations of a human burial from the Yuha Desert, California". American Antiquity. Vol. 43, no. 3.

PAYEN, Louis A. et al. 1979. "Reply to Bischoff, Childers and Shlemon". American Antiquity. Vol. 44, no. 3.

REEVES, B.O.K. 1985. "Pleistocene Archaeological Research in San Diego". Current Research in the Pleistocene. Vol. 2. 
RITTER, Eric W. 1976. "The Antiquity of Man in the Laguna Seca Chapala Basin of Baja California". Pacific Coast Archaeological Society Quarterly. Vol. 12, no. 1.

- 1979a. "Archaeological Investigations at Laguna Seca Chapala, Estado de Baja California, México". (Mimeo).

- 1979b. "An Archaeological Study of South-central Baja California, México". Tesis Doctoral, University of California, Davis.

RITTER, Eric W., Louis A. Payen, y Carol Huber Rector. 1978. “A Brief Note on An Archaeological Reconnaissance to Laguna la Guija, Baja California". Pacific Coast Archaeological Society Quarterly. Vol. 14, no. 1.

- 1984. "An Archaeological Survey of Laguna la Guija, Baja California”. Pacific Coast Archaeological Society Quarterly. Vol. 20, no. 1.

ROGERS, Malcolm J. 1939. "Early Lithic Industries of the Lower Basin of the Colorado River and Adjacent Desert Areas". San Diego Museum Papers. No.3.

- 1945. "An Outline of Yuman Prehistory". Southwestern Journal of Anthropology. Vol. 1.

- 1966. Anclent Hunters of the Far West. Union-Tribune, San Diego. SHIPMAN, P. 1981. Life History of a Fossil: An Introduction to Taphonomy and Paleoecology. Harvard University Press, Cambridge.

STAFFORD, T.W., Jr. et al. 1984. "Holocene Age of the Yuha Burial: Direct Radiocarbon Determinations by Accelerator Mass Spectrometry". Nature. No. 308.

TREGANZA, Adan E. 1942. "An Archaeological Reconnaissance of Northeastern Baja California and Southeastern California". American Antiquity. Vol. 8, no. 1.

- 1947. "Notes on the San Dieguito lithic industry of southern California and northem Baja California". University of California Publications in American Archaeology and Ethnology. No. 44.

WARREN, Claude N. 1966. "The San Dieguito type site: M.J. Rogers' 1938 Excavation on the San Dieguito River". San Diego Museum Papers. No.6.

- 1967. "The San Dieguito Complex: A Review and Hypothesis". American Antiquity. Vol. 32, no. 1.

WARREN, Claude N. y Robert H. Crabtree. 1986. "Prehistory of the Southwestern Area". En: Warren L. D'Azevedo, (ed.) Great Basin, Handbook of North American Indians. Smithsonian Institution, Washington, D.C.

WARREN, Claude N. y A.J. Ranere. 1968. "Outside Danger Cave: A View of Early Man in the Great Basin". Eastern New Mexico University Contributions in Anthropology. No. 1. 
WARREN, Claude N. y Delbert L. True. 1961. "The San Dieguito Complex and its Place in California Prehistory". University of California, Los Angeles, Archaeological Survey Annual Report 1962-1963.

WILKE, Philip J. 1978. "Cairn Burials of the California Deserts". American Antiquity. Vol. 43, no. 3. 